\title{
UNIQUENESS OF COMMUTING COMPACT APPROXIMATIONS
}

\author{
BY
}

\author{
RICHARD B. HOLMES, BRUCE E. SCRANTON AND JOSEPH D. WARD
}

ABSTRACT. Let $H$ be an infinite dimensional complex Hilbert space, and let $B(H)$ (resp. $C(H)$ ) be the algebra of all bounded (resp. compact) linear operators on $H$. It is well known that every $T \in \mathscr{B}(H)$ has a best approximation from the subspace $\mathcal{C}(H)$. The purpose of this paper is to study the uniqueness problem concerning the best approximation of a bounded linear operator by compact operators. Our criterion for selecting a unique representative from the set of best approximants is that the representative should commute with $T$. In particular, many familiar operators are shown to have zero as a unique commuting best approximant.

Introduction. Let $H$ be an infinite dimensional complex Hilbert space, and let $\mathcal{B}(H)$ (resp. $\mathcal{C}(H)$ ) be the algebra of all bounded (resp. compact) linear operators on $H$. It is well known [4], [6] that $\mathcal{C}(H)$ is proximinal in $B(H)$, that is, for every $T \in B(H)$ there exists a $C \in \mathcal{C}(H)$ such that $\|T-C\|=$ $\operatorname{dist}(T, \mathcal{C}(H))$. It was shown, in [7], for arbitrary noncompact $T$ that the set $\mathcal{P}(T)$ of best compact approximants to $T$ has infinite dimension. From this proposition it can be deduced that $c_{0}$ viewed as a subspace of $m$ has the same property. These spaces are the first "natural" proximinal subspaces known to the authors to have such a property. This phenomenon leads one to the question of finding a unique representative from $\mathcal{P}(T)$. Thus the purpose of this paper is to study the uniqueness problem concerning the best approximation of a bounded linear operator by compact operators. Our criterion for selecting a unique representative $C_{T}$ from $\mathcal{P}(T)$ is that $C_{T}$ should commute with $T$.

Now, in general, to satisfy our criterion for arbitrary $T$ is not an easy task, since Lomonosov has shown [8] that any operator commuting with a nontrivial compact operator has a nontrivial invariant subspace. However, we recall from [7] that operators in the set $\mathcal{C}(H)^{0} \equiv\{T \in \mathfrak{B}(H) \mid\|T\|=$ $\operatorname{dist}(T, \mathcal{C}(H))\}$ (anticompact operators) have, by definition, a commuting best compact approximant, namely 0 . The anticompact operators have been considered by Coburn [2] and were termed "extremely noncompact." To study

Received by the editors August 17, 1973 and, in revised form, June 5, 1974. AMS (MOS) subject classifications (1970). Primary 41A50, 41A65, 47B05; Secondary 47A30, 47B20, 47D20. 
this situation in more detail, we introduce two classes of operators in $B(H)$ : $Z U C=\{T \in \mathscr{S}(H I) \mid 0$ is the unique compact operator that coinmutes with $T\}$ and

$Z U C A=\{T \in B(H) \mid 0$ is the unique operator in $\mathcal{P}(T)$ that commutes with $T\}$. Clearly, ZUC $\cap \mathcal{C}(H)^{0} \subset Z U C A \subset \mathcal{C}(H)^{0}$ and, as we shall see, these inclusions are proper. The following fact, whose proof is omitted, constitutes the only general necessary condition known to us for membership in the classes ZUC or ZUCA.

Proposition 1. An operator in $\beta(H)$ cannot belong to ZUC or ZUCA if it has a compact direct summand.

In the first section of this paper we show that several classes of operators are in $Z U C A$ be virtue of being in $Z U C \cap \mathcal{C}(H)^{0}$. In the second section we provide criteria for a weighted shift to belong to the various operator classes $\mathcal{C}(H)^{0}, Z U C$, and ZUCA. In the final two sections we consider some counterexamples and open questions. Any terms not defined in this paper may be found in [5].

At this time we would like to thank Professor C. R. Putnam for his many helpful discussions.

1. Operators in $Z U C \cap \mathcal{C}(H)^{0}$. What sort of operators are in ZUCA? Many operators are in $Z U C A$ by virtue of being in $Z U C \cap \mathcal{C}(H)^{0}$. We begin the investigation of this latter subset by identifying a large class of operators in $\mathcal{C}(H)^{0}$.

Let $r_{e}(T)$ be the essential spectral radius of $T \in \mathfrak{B}(H)$. Although there are several notions of essential spectrum, it was shown in [9] that the corresponding essential spectral radii are all the same. Hence $r_{e}(T)$ is unambiguously defined as, for example, $\max \left\{|\lambda| \mid \lambda \in \bigcap_{C \in \mathcal{C}(H)}\right.$ Spectrum $\left.(T+C)\right\}$.

Definition. $T \in \mathscr{B}(H)$ is essentially normaloid if $r_{e}(T)=\|T\|$.

In [7] it was observed that seminormal operators with empty point spectrum are essentially normaloid, and the following proposition was proved:

Proposition 2. Every essentially normaloid operator is anticompact.

Our strategy for this section may now be described. We will use Proposition 1 to restrict our attention to certain essentially normaloid operators. Then, in view of Proposition 2, to prove that such an operator is in ZUCA it suffices to show that the operator belongs to $Z U C$. 
Theorem 1. A normal operator is in $Z U C \cap \mathcal{C}(H)^{0}$ if and only if its point spectrum is empty.

Proof. Since any eigenspace of a normal operator is a reducing subspace, a normal operator with an eigenvalue has a compact direct summand and by Proposition 1 is not in ZUCA.

Conversely, let $N$ be a normal operator with empty point spectrum. By the preceding discussion it is sufficient to show that $N \in Z U C$. Suppose that $C$ is a compact operator and $C$ commutes with $N$ (written $C \leftrightarrow N$ ). We show $C=0$. Now $N \leftrightarrow C$ implies $N \leftrightarrow C^{*}$ (Fuglede's theorem). Thus $N$ $\leftrightarrow C$ implies $N \leftrightarrow C^{*} C$. Since $C^{*} C$ is a positive, compact operator, the Schmidt (polar) decomposition asserts that the spectrum of $C^{*} C$ consists of 0 and a (possibly empty) decreasing sequence of positive eigenvalues, each of finite multiplicity.

Suppose that $E$ is an eigenspace of $C^{*} C$ corresponding to a positive eigenvalue. It is easy to check that $N \leftrightarrow C^{*} C$ implies $E$ is an invariant subspace of $N$. Since $E$ is finite dimensional, this means that $N$ must have an eigenvalue, which contradicts our hypothesis. Thus the spectrum of $C^{*} C$ is $\{0\}$. Hence $C^{*} C=0$, which implies $C=0$. Q.E.D.

Theorem 2. An isometry is in $Z U C \cap \mathcal{C}(H)^{0}$ if and only if its point spectrum is empty.

Proof. Express the isometry in its Wold decomposition [5] as $U \oplus W$, where $U$ is a pure isometry (i.e. a unilateral shift of some multiplicity) and $W$ is a unitary operator. Any eigenspace of the isometry must be an eigenspace of the unitary part, and hence a reducing subspace of the isometry. Thus if an isometry has an eigenvalue, it has a compact direct summand, and by Proposition 1 it is not in ZUCA.

Conversely, if the point spectrum of the isometry (a subnormal operator) is empty, Proposition 2 is applicable, and it is sufficient to show that the isometry is in ZUC.

First, consider a pure isometry $U . U$ is defined by

$$
U\left(x_{1}, x_{2}, x_{3}, \ldots\right)=\left(0, x_{1}, x_{2}, x_{3}, \ldots\right)
$$

where the $x_{j}$ are elements of a fixed Hilbert space $K$ such that $\Sigma\left\|x_{j}\right\|^{2}<$ $\infty_{\text {. Let }} x \in K$ be a fixed unit vector, and define $e_{n}=(0, \ldots, 0, x, 0, \ldots)$ where $x$ is the $n$th component of $e_{n}$. Then $\left\{e_{n}\right\}_{n=1}^{\infty}$ is an orthonormal sequence in the domain of $U$. Suppose $C$ is a compact operator and $C \leftrightarrow U$. Then 
which implies

$$
U C\left(e_{n}\right)=C U\left(e_{n}\right)=C\left(e_{n+1}\right)
$$

$$
\cdots=\left\|C\left(e_{n+1}\right)\right\|=\left\|C\left(e_{n}\right)\right\|=\cdots=\left\|C\left(e_{1}\right)\right\| .
$$

Because $C$ is compact, $\lim C\left(e_{n}\right)=0$, and hence $C\left(e_{n}\right)=0$ for every $n$; that is, $C=0$.

Consider any compact operator $\hat{C}$ which commutes with the isometry. Corresponding to the Wold decomposition $\left[\begin{array}{ll}U & 0 \\ 0 & W\end{array}\right]$ of the isometry we have $\hat{C}=$ $\left[\begin{array}{ll}A & B \\ C & D\end{array}\right]$, where $A, B, C$, and $D$ are compact. From the commutativity of these operators it follows that $A \leftrightarrow U$ and $D \leftrightarrow W$, so by the above paragraph and Theorem 1 we have $A=0$ and $D=0$. Further, $C U=W C$, and if we consider $e_{n}$ as above we have

$$
W C\left(e_{n}\right)=C U\left(e_{n}\right)=C\left(e_{n+1}\right)
$$

and $\cdots=\left\|C\left(e_{n+1}\right)\right\|=\left\|C\left(e_{n}\right)\right\|=\cdots=\left\|C\left(e_{1}\right)\right\|$. As before, the compactness of $C$ implies that $C=0$. Lastly, $B W=U B$, so that $W^{*} B^{*}=B^{*} U^{*}$. Again letting $e_{n}$ be as above, and recalling that $U^{*}$ is the backwards shift we have

$$
W^{*} B^{*}\left(e_{n+1}\right)=B^{*} U^{*}\left(e_{n+1}\right)=B^{*}\left(e_{n}\right)
$$

and

$$
\cdots=\left\|B^{*}\left(e_{n+1}\right)\right\|=\left\|B^{*}\left(e_{n}\right)\right\|=\cdots=\left\|B^{*}\left(e_{1}\right)\right\|=0,
$$

so that $B^{*}=0$, whence $B=0$. Q.E.D.

Before proceeding to the last classes of operators in $Z U C \cap \mathcal{C}(H)^{0}$, we state and prove a proposition that will be used to show that the operators are in $Z U C$. The fact that $Z U C$ and $Z U C A$ are invariant under adjunction is easy to verify and is used in the proposition.

Proposition 3. If an operator has empty point spectrum and its adjoint has so many simple eigenvalues that the corresponding eigenvectors are fundamental in $H$, then the adjoint of the operator (hence the operator itself) is in ZUC.

Proof. Suppose $C \leftrightarrow T$ and $C$ is compact. By an argument similar to the one used in the proof of Theorem 1 , it is clear that spectrum $(C)=\{0\}=$ spectrum $\left(C^{*}\right)$. We show that $C^{*}=0$ by showing $C^{*}(x)=0$ for any eigenvector $x$ associated with a simple eigenvalue $\lambda$ of $T^{*}$. Since $C \leftrightarrow T$, we have $C^{*} \leftrightarrow T^{*}$ so that $T^{*} \dot{C}^{*}(x)=C^{*} T^{*}(x)=\lambda C^{*}(x)$. Since $\lambda$ is a simple 
eigenvalue of $T^{*}, x$ must be an eigenvector of $C^{*}$. Because spectrum $\left(C^{*}\right)$ $=\{0\}$, we have $C^{*}(x)=0$. Q.E.D.

Theorem 3. Each of the following (classes of) operators is contained in $Z U C \cap \mathcal{C}(H)^{0} \subset Z U C A$ :

(a) the discrete Cesaro operator,

(b) multiplication by a bounded schlicht function on some Bergman space,

(c) Toeplitz operators whose corresponding multiplication function is schlicht.

Proof. It is well known that these operators are subnormal and have empty point spectrum; thus, in accord with the strategy of this section, it is sufficient to show that they belong to ZUC. This we will do by showing that in each of these cases the hypotheses of Proposition 3 are satisfied.

Proof of (a). In [1] the following facts were proved: the point spectrum of the adjoint of the discrete Cesaro operator is $\{\lambda|| 1-\lambda \mid<1\}$; each of these eigenvalues is simple; when $l^{2}$ is identified with the Hardy space $H^{2}$ in the natural manner, the function $(1-z)^{1 / \lambda-1}$ is an eigenvector associated with $\lambda$. It remains to show that these eigenvectors are fundamental. By considering $\lambda=1,1 / 2,1 / 3, \cdots$ it is easy to see that the span of the eigenvectors includes $1, z, z^{2}, \ldots$. Thus the span of the eigenvectors of the adjoint of the discrete Cesaro operator is dense. Q.E.D.

Proof of (b). Let

$D=$ a fixed region in the complex plane,

$\phi=$ a bounded schlicht function on $D$,

$T=$ multiplication by $\phi$ on $A^{2}(D)$,

$K_{\lambda}=$ reproducing element for "evaluation at $\lambda$ " functional $\delta_{\lambda}$. Since $\left\{K_{\lambda}\right\}_{\lambda \in D}$ is fundamental in $A^{2}(D)$, it is sufficient to show that $\overline{\phi(\lambda)}$ is a simple eigenvalue of $T^{*}$ with corresponding eigenvector $K_{\lambda}$, for each $\lambda \in D$. To do this recall that

$$
\operatorname{ker}\left(T^{*}-\overline{\phi(\lambda)} I\right)=\operatorname{ran}(T-\phi(\lambda) I)^{\perp} .
$$

Thus, using the definition of $K_{\lambda}$, it is easy to check that $K_{\lambda}$ is an eigenvector associated with $\overline{\phi(\lambda)}$. To see that $\overline{\phi(\lambda)}$ is simple we verify that $\operatorname{ran}(T-\phi(\lambda) I)$ is the kernel of a linear functional, viz.,

$$
\operatorname{ran}(T-\phi(\lambda) I)=\left\{g \in A^{2}(D) \mid g(\lambda)=0\right\}=\operatorname{ker}\left\{\delta_{\lambda}\right\}
$$

Now we clearly have 


$$
\begin{aligned}
\operatorname{ran}(T-\phi(\lambda) I) & =\left\{g \mid g(z)=(\phi(z)-\phi(\lambda)) f(z) \text { for some } f \in A^{2}(D)\right\} \\
& \subset\left\{g \in A^{2}(D) \mid g(\lambda)=0\right\} .
\end{aligned}
$$

For any $g \in A^{2}(D)$ such that $g(\lambda)=0$ we may define $f(z)=g(z) /(\phi(z)-\phi(\lambda))$, and the problem reduces to showing $f \in A^{2}(D)$. $f$ is defined at $z=\lambda$ since

$$
\lim _{z \rightarrow \lambda} \frac{g(z)}{\phi(z)-\phi(\lambda)}=\frac{g^{\prime}(\lambda)}{\phi^{\prime}(\lambda)}
$$

and $\phi^{\prime}(\lambda) \neq 0$ because $\phi$ is schlicht [11, p. 198]. It is similarly easy to check that $f$ is differentiable at $z=\lambda$. To see that $f \in L^{2}(D)$, note that $f$ is continuous on a disc $D_{\lambda}$ centered at $\lambda$ and contained in $D$. Thus $f$ is certainly in $L^{2}\left(D_{\lambda}\right)$. It suffices to show that $|\phi(z)-\phi(\lambda)|$ is bounded away from 0 on $D \backslash D_{\lambda}$. If this were not true, there would exist $z_{n}, n=1,2, \ldots$, in $D \backslash D_{\lambda}$ such that $\phi\left(z_{n}\right) \rightarrow \phi(\lambda)$ as $n \rightarrow \infty$. Since $\phi^{-1}$ is also analytic on $D$ [11, p. 199], $z_{n} \rightarrow \lambda$ as $n \rightarrow \infty$. This is a contradiction. Q.E.D.

Proof of (c). Using the representation of the Hardy space as $H^{2}(D)$ where $D$ is the open unit disc, the proof is essentially the same as in part (b). The only difference is that for $g \in H^{2}(D)$ such that $g(\lambda)=0$, it must be observed that $\int_{0}^{2 \pi}\left|f\left(r e^{i \theta}\right)\right|^{2} d \theta$ is uniformly bounded for $r$ sufficiently close to but less than 1 , where $f(z)=g(z) /(\phi(z)-\phi(\lambda))$. The proof of this observation is also analogous to the corresponding one in part (b). Q.E.D.

Remark 1. The above classes of operators in ZUC (ZUCA) are all hyponormal (even subnormal) and have empty point spectrum. From Proposition 1 and the fact that eigenspaces reduce hyponormal operators it follows that the empty point spectrum assumption was necessary for such operators to be in ZUC (ZUCA). However, this necessary condition breaks down for seminormal operators. For example, the adjoint of the unilateral shift is in $Z U C$ (and $Z U C A$ ) by Proposition 2; yet its point spectrum is the open unit disc.

Remark 2. Although the result of Shields and Wallen [10, Theorem 2] implies that their multiplication operators $M_{z}$ belong to $Z U C$, Proposition 3 is applicable to a more general situation where their condition (c) is significantly weakened and condition (d) is eliminated. We also mention that Theorem 3(c) has recently been proved independently by Deddens and Wong [3].

2. Weighted shifts. In this section we consider the following question: Which weighted shifts belong to the classes $\mathcal{C}(H)^{0}, Z U C$, and $Z U C A$ ? We will use the following notation for a weighted shift throughout this section:

$$
T=\sum_{n=1}^{\infty} \alpha_{n} e_{n+1} \otimes \bar{e}_{n}
$$


i.e.

$$
T(x)=\sum_{n=1}^{\infty} \alpha_{n}\left\langle x, e_{n}\right\rangle e_{n+1}
$$

where $\left\{e_{n}\right\}_{n=1}^{\infty}$ is an orthonormal basis of $H$, chosen in such a way that the weights $\alpha_{n}$ are nonnegative. If $T$ had a zero weight it would have a finite rank direct summand, and by Proposition 1 it would not be in ZUC or ZUCA. Hence, we will require that all the weights be positive.

We begin by characterizing the weighted shifts in $Z U C$. For $T \in \mathcal{B}(H)$ a necessary condition for $T$ to be in $Z U C$ is that $T^{n}$ be noncompact for every positive integer $n$. It is interesting to note that for weighted shifts this condition is also sufficient.

Theorem 4. A weighted shift $T$ with positive weights $\alpha_{n}$ belongs to ZUC if and only if there does not exist a $k_{0}>1$ so that $\lim _{n}\left(a_{n+1} \cdots a_{n+k_{0-1}}\right)$ $=0$.

Proof. If there exists $k_{0}>1$ such that $\lim _{n}\left(a_{n+1} \cdots a_{n+k_{0}-1}\right)=0$, then by the Schmidt decomposition $T^{k_{0-1}}$ is compact and $T$ is not in ZUC.

Conversely, suppose $C \leftrightarrow T$. This is equivalent to

$$
T C\left(e_{n}\right)=C T\left(e_{n}\right)=a_{n} C\left(e_{n+1}\right) \text { for all } n \text {. }
$$

Hence

$$
C\left(e_{n+1}\right)=\frac{T}{a_{n}} C\left(e_{n}\right)=\cdots=\frac{T^{n}}{a_{n} \cdots a_{1}} C\left(e_{1}\right) \text { for all } n \text {. }
$$

If $T$ is not in $Z U C$, then we may assume that the above $C$ is compact and nonzero. Thus $C\left(e_{1}\right) \neq 0$ and we may write $C\left(e_{1}\right)=\sum_{j=k_{0}}^{\infty} \beta_{j} e_{j}$ with $\beta_{k_{0}} \not$ 0. Because $\left\|T^{n}\left(C\left(e_{1}\right)\right)\right\| \geq\left|\beta_{k_{0}} \alpha_{k_{0}} \cdots \alpha_{n+k_{0}-1}\right|$, and $C$ is compact we have

$$
\begin{aligned}
0 & =\lim _{n}\left\|C\left(e_{n+1}\right)\right\|=\lim _{n} \frac{1}{a_{n} \cdots a_{1}}\left\|T^{n}\left(C\left(e_{1}\right)\right)\right\| \\
& \geq \lim _{n}\left|\beta_{k_{0}}\right| \frac{a_{k_{0}} \cdots a_{n+k_{0}-1}}{\alpha_{n} \cdots a_{1}}=\frac{\left|\beta_{k_{0}}\right|}{a_{k_{0}-1} \cdots a_{1}} \lim _{n} \alpha_{n+1} \cdots a_{n+k_{0}-1} .
\end{aligned}
$$

Hence from the term immediately after the inequality we see that $k_{0}>1$, and it follows that $\lim _{n}\left(a_{n+1} \cdots a_{n+k_{0-1}}\right)=0$. Q.E.D.

The following remarks will be useful later, and refer to a weighted shift $T$ with positive weights. 
Remark 3. If $T \notin \mathcal{C}(H)$ and $T \notin Z U C$, then the $k_{0}$ in this theorem satisfies $k_{0} \geq 3$.

Remark 4. If $0 \neq C \in \mathcal{C}(H)$ and $C \leftrightarrow T$, then $C\left(e_{1}\right)=\sum_{j=k_{0}}^{\infty} \beta_{j} e_{j}$ with $\beta_{k_{0}} \neq 0$ and $k_{0}>1$. Thus for $n \geq 1$,

$$
C\left(e_{n+1}\right)=\frac{T^{n}}{\alpha_{n} \cdots \alpha_{1}} C\left(e_{1}\right)=\frac{1}{\alpha_{n} \cdots \alpha_{1}} \sum_{j=k_{0}}^{\infty} \beta_{j}\left(\alpha_{j} \cdots a_{j+n-1}\right) e_{j+n},
$$

so that $C\left(e_{n+1}\right)$ is orthogonal to $e_{1}, \ldots, e_{n+k_{0-1}}$.

Remark 5. If $C \in \mathcal{C}(H)$ and $C \leftrightarrow T$, then $C=0$ if and only if $C\left(e_{n}\right)=$ 0 , for some integer $n$.

In [7] a characterization of the weighted shifts with nonnegative weights in $\mathcal{C}(H)^{0}$ was given, namely:

Proposition 4. A weighted shift with nonnegative weights $a_{n}$ belongs to $\mathcal{C}(H)^{0}$ if and only if $\sup _{n} \alpha_{n}=\lim \sup _{n \rightarrow \infty} a_{n}$.

Thus combining Propositions 3 and 4, we obtain a characterization of all weighted shifts in $Z U C \cap \mathcal{C}(H)^{0}$ in terms of the weights. From this characterization it may be easily verified that

Corollary. A hyponormal weighted shift is in $Z U C \cap \mathcal{C}(H)^{0}$ if and only if its point spectrum is empty.

We do not know a necessary and sufficient condition for a weighted shift to be in ZUCA. We do know, however, that the weighted shifts in ZUC $\cap$ $\mathcal{C}(H)^{0}$ do not exhaust the weighted shifts in $Z U C A$. The next proposition will enable us to exhibit such an example.

Proposition 5. Let $T \in \mathcal{C}(H)^{0}$ be a weighted shift (with positive weights) which attains its norm. Then $T \in Z U C A$.

Proof. Let $m$ be an integer such that $a_{m}=\|T\|$. Suppose that $0 \neq C \epsilon$ $\mathcal{P}(T)$ and $C \leftrightarrow T$. Then

$$
\|T\|^{2}=\|T-C\|^{2} \geq\left\|(T-C)\left(e_{m}\right)\right\|^{2}=\left\|\alpha_{m} e_{m+1}-C\left(e_{m}\right)\right\|^{2} .
$$

By Remark 3, $k_{0} \geq 3$, so, by Remark 4, $e_{m+1}$ is orthogonal to $C\left(e_{m}\right)$. So $\|T\|^{2} \geq a_{m}^{2}+\left\|C\left(e_{m}\right)\right\|^{2}$, whence $C\left(e_{m}\right)=0$. Thus, by Remark $5, C=0$. This proves that $T \in Z U C A$.

Remark 6. We now use this proposition to prove that the inclusion ZUC $\cap \mathcal{C}(H)^{0} \subset Z U C A$ is proper. Consider the operator

$$
T=\sum_{n \text { odd }} e_{n+1} \otimes \overline{e_{n}}+\sum_{n \text { even }} \frac{1}{n} e_{n+1} \otimes \bar{e}_{n} .
$$


From Proposition 4 and Theorem 4 it follows that $T \in \mathcal{C}(H)^{0}$ and $T \notin Z U C$. However, Proposition 5 is satisfied so $T \in Z U C$.

The condition, in Proposition 5, that $T$ attain its norm may be relaxed to the condition that a subsequence of the weights approaches the norm relatively quickly. To be more precise, suppose for $T \notin Z U C$ we let $k_{0}$ be the smallest integer so that $k_{0}>1$ and the condition of Theorem 4 is satisfied. Then we have

Proposition 6. If $T$ is a weighted shift with positive weights, $T \in \mathcal{C}(H)^{0}$, $T \notin Z U C$, and for every $\beta>0$ and $k \geq k_{0}$ there exists an $m$ depending upon $\beta$ and $k$ so that

$$
\|T\|^{2}-\alpha_{m+1}^{2}<\left(\beta \alpha_{k} \cdots \alpha_{m+k-1}^{2}\right) /\left(\alpha_{m} \cdots \alpha_{1}\right),
$$

then $T \in Z U C A$.

The proof is omitted since its essence is contained in the proof of Proposition 5. This result enlarges the class of weighted shifts known to be in ZUCA.

3. A counterexample. It has been established that all normal operators with empty point spectrum and several other classes of hyponormal operators with empty point spectrum are in $Z U C \cap \mathcal{C}(H)^{0} \subset Z U C A$. One might suspect that all hyponormal operators with empty point spectrum are in ZUCA. This is decidedly not the case as is demonstrated by the following proposition and its corollary.

Proposition 7. There exists a quasinormal operator with empty point spectrum having a nonzero commuting compact operator.

Proof. Let $H=l^{2},\left\{e_{n}\right\}_{n=1}^{\infty}$ the standard orthonormal basis in $l^{2}$, and define $P_{0}(x)=\sum_{n=1}^{\infty} \alpha_{n} x_{n} e_{n}$ where $x=\sum_{n=1}^{\infty} x_{n} e_{n}$ and $a_{n}>a_{n+1}>0$ for all $n . P_{0}$ is a positive operator on $l^{2}$. Let $T=U P$ be the dilated shift operator defined by $P_{0}$, i.e., $\operatorname{dom}(T)=\bigoplus_{1}^{\infty} H_{j}, H_{j}=H, P=\bigoplus_{1}^{\infty} P_{j}, P_{j}=P_{0}$, and $U$ = unilateral shift on $\bigoplus_{1}^{\propto} H_{j}$. Now the point spectrum of $T$ is empty since $P_{0}$ is injective, and $U P=P U$, so $T$ is quasinormal.

We recall the Rellich criterion for compact operators: an operator $C$ is compact if and only if for any $\epsilon>0$ there exists a finite codimensional subspace $V_{\epsilon}$ such that $\left\|C \mid V_{\epsilon}\right\| \leq \epsilon_{\text {. Let }} C_{j} \in \mathcal{C}(H)$. The Rellich criterion implies that $C=\bigoplus_{1}^{\infty} C_{j}$ defined on $\bigoplus_{1}^{\infty} H_{j}$ is compact if and only if $\left\|C_{j}\right\| \rightarrow 0$ as $j \rightarrow \infty$. It is also easy to verify that $C \leftrightarrow T$ if and only if

$$
P_{0} C_{j}=C_{j+1} P_{0} \text { for all } j \text {. }
$$


So it suffices to make a choice of $C_{j}$ satisfying these equations and such that $\left\|C_{j}\right\| \rightarrow 0$.

Define $C_{j}=\Sigma_{n=1}^{\infty} \beta_{n}^{(j)} e_{n+1} \otimes \bar{e}_{n}$, where $\beta_{n}^{(1)} \downarrow 0$ as $n \rightarrow \infty$ and $\beta_{n+1}^{(j)}=$ $\beta_{n+1}^{(j-1)} \alpha_{n+1} / \alpha_{n}$. Let us now require that $\sup _{n}\left(\alpha_{n+1} / \alpha_{n}\right)=A<1$ (e.g., $\alpha_{n}$ $\left.=2^{-(n+1)}\right)$. Then $\beta_{n+1}^{(j)} \leq A^{j-1} \beta_{n+1}^{(1)}<A^{j-1} \beta_{1}^{(1)}$, and since $\left\|C_{j}\right\|=\sup _{n} \beta_{n}^{(j)}$

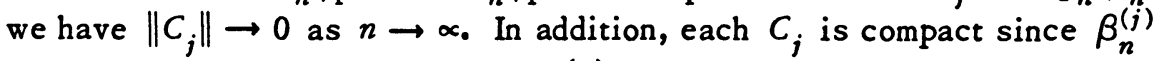
$\rightarrow 0$ as $n \rightarrow \infty$. Finally, condition (*) is satisfied so that $C \leftrightarrow T$.

Corollary. There exists a quasinormal operator with empty point spectrum having a nonzero commuting compact best approximant.

Proof. Let $T$ and $C$ be as in the previous example, let $N$ be a normal operator with empty point spectrum on some Hilbert space, and consider the operator $N \oplus T$, on the appropriate Hilbert space $\mathcal{H} . N \oplus T$ is a quasinormal operator, and its point spectrum is empty. Suppose that $\|N\|>\|T-C\|$ and $\|N\|>\|T\|$. In [7] it was proved that if $C_{1}$ is a best compact approximant to $N$ and $C_{2}$ is a best compact approximant to $T$, then

$$
\operatorname{dist}(N \oplus T, \mathcal{C}(\mathcal{H}))=\left\|N \oplus T-C_{1} \oplus C_{2}\right\| \text {. }
$$

Because 0 is a best compact approximant to $N$ (by Proposition 2) and $\left\|T-C_{2}\right\| \leq\|T\|<\|N\|$, it follows that

$$
\operatorname{dist}(N \oplus T, \mathcal{C}(\mathcal{H}))=\max \left\{\|N\|,\left\|T-C_{2}\right\|\right\}=\|N\| \text {. }
$$

Thus if we let $K=0 \oplus C \neq 0$, we see that $K \leftrightarrow N \oplus T$ and

$$
\|N \oplus T-K\|=\|N\|=\operatorname{dist}(N \oplus T, \mathcal{C}(\mathcal{H})) \text {. Q.E.D. }
$$

4. The discontinuous nature of $Z U C(Z U C A)$. The relationship between the metric complement $\mathcal{C}(H)^{0}$ and its subsets $Z U C A$ is interesting. For example, the possibility that ZUCA is dense in $\mathcal{C}(H)^{0}$ is an intriguing but open question. However, neither ZUCA nor ZUC is closed.

Proposition 8. There is a sequence of selfadjoint operators with empty point spectrum that converges to the identity operator.

Proof. Let $S$ be any selfadjoint operator with empty point spectrum. Evidently $T_{n}=I+\epsilon_{n} S, \epsilon_{n} \rightarrow 0$, is a sequence of selfadjoint operators with empty point spectrum converging uniformly to $I$. Q.E.D.

By Theorem 1, the $T_{n}$ 's are in ZUC and ZUCA; however, $I$ is in neither. Such a phenomenon illustrates the delicate and discontinuous nature of the ZUCA property since we have just exhibited a sequence of operators each of whose set of commuting best compact approximations is zero dimensional, 
but whose (norm) limit has an infinite dimensional set of commuting best compact approximations.

\section{REFERENCES}

1. A. Brown, P. R. Halmos and A. L. Shields, Cesàro operators, Acta Sci. Math. (Szeged) 26 (1965), 125-137. MR 32 \#4539.

2. L. A. Coburn, Weyl's theorem for nonnormal operators, Michigan Math. J. 13 (1966), 285-288. MR 34 \#1846.

3. J. Deddens and T. Wong, The commutant analytic Toeplitz operators, Trans. Amer. Math. Soc. 184 (1973), 261-273. MR 48 \#2819.

4. I. C. Gohberg and M. G. Krein, Introduction to the theory of linear nonselfadjoint operators in Hilbert space, "Nauka", Moscow, 1965; English transl., Transl. Math. Monographs, vol. 18, Amer. Math. Soc., Providence, R. I., 1969. MR 36 \#3137; 39 \#7447.

5. P. R. Halmos, A Hilbert space problem book, Von Nostrand, Princeton, N. J., 1967. MR $34 \# 8178$.

6. R. Holmes and B. Kripke, Best approximation by compact operators, Indiana Univ. Math. J. 21 (1971), 255-263. MR 45 \#5718.

7. R. Holmes, B. Scranton and J. Ward, Approximation from the space of compact operators and other M-ideals, Duke Math. J. (to appear).

8. V. Lomonosov, Invariant subspaces for the family of operators which commute with a completely continuous operator, Funkcional. Anal. i Priložen. 7 (1974), 213214. (Russian)

9. R. D. Nussbaum, The radius of the essential spectrum, Duke Math. J. 37 (1970), 473-478. MR 41 \#9028.

10. A. L. Shields and L. J. Wallen, The commutants of certain Hilbert space ope rators, Indiana Univ. Math. J. 20 (1970/71), 777-788. MR 44 \#4558.

11. E. C. Titchmarsh, The theory of functions, 2nd ed., Oxford Univ. Press, Oxford, 1939.

DIVISION OF MATHEMATICAL SCIENCES, PURDUE UNVERSITY, LAFAYETTE, INDIANA (Current address of R. B. Holmes)

Current address (B. E. Scranton): Daniel H. Wagner, Associates, Paoli, Pennsylvania 19301

Current address (J.D. Ward): Department of Mathematics, Texas A \& M University, College Station, Texas 77843 